\title{
Donor specific antibody-mediated rejection in allogeneic hematopoietic stem cell transplant recipients
}

\begin{abstract}
Lack of engraftment owing to preformed donor specific antibodies (DSAs) is a serious complication of hematopoietic stem cell transplantation (HSCT). Identification of patients at risk is critical to improve outcome. In this review, current clinical approaches are discussed with emphasis on methods of detecting DSAs and preventive measures. DSA-specific mean fluorescence intensity measurements by flow cytometry are a reliable, relatively easy and fast approach to quantify antibody levels. Preventive measures used to decrease DSAs prior to HSCT include IVIG, plasmapheresis, Rituximab, T cell depleting agents, bortezomib, eculizumab and splenectomy. As of today, there isn't a single standardized approach to reduction of DSAs prior to HSCT. Various institutions have borrowed mainly from their solid organ transplant (SOT) experience and have come up with multipronged approaches. However, the superiority of one method vs another remains to be established.
\end{abstract}

Keywords: donor specific antibodies (DSAs), hematopoietic stem cell transplantation (HSCT), graft rejection, engraftment failure
Volume I Issue 3 - 2015

\author{
Zartash Gul, Gerhard Hildebrandt \\ Division of Hematology \& BMT, University of Kentucky, USA
}

Correspondence: Gerhard Hildebrandt, Division of Hematology \& BMT, University of Kentucky, Lexington, KY, 40536,Tel 80I-585-2626, Email Gerhard.hildebrandt@uky.edu

Received: June 29, 2015 | Published: July 17, 2015

\section{Mini review}

Graft rejection remains a significant concern after hematopoietic stem, cell transplantation (HSCT). While its incidence and predisposing factors are well known in the setting of solid organ transplantation (SOT), the study of graft rejection still remains an area of active investigation in case of HSCT. Recipient T cells, natural killer cells and antibodies are implicated in graft rejection. Other factors that can contribute towards it are HLA mismatch, reduced intensity conditioning and hematopoietic stem cell dose. ${ }^{1}$

With the increasing use of haploidentical transplants there is an increasing risk of antibody mediated graft rejection, a scenario that as mentioned above was more common in case of SOT. Studies have shown that while $\mathrm{T}$ cell mediated rejection can take upto 6days to come into effect, preformed antibodies can lead to rapid rejection in less than 3hours. ${ }^{2}$ Donor specific antibodies (DSA) can lead to graft rejection in the setting of both solid organ (SOT) and hematopoietic stem cell transplant (HSCT). ${ }^{3}$ The issue has been studied more in depth in case of SOT and the experience and protocols that resulted have been borrowed and implemented in HSCT.

DSA can be detected by various means. However, flow cytometry assays remain one of the most commonly used methods and the signal strength reflective of DSA levels is denoted by mean fluoresce intensity (MFI) ${ }^{4}$ It is a question of some debate, whether there is a certain quantitative DSA level being significant enough to cause rejection or below which someone can feel entirely safe. A common practice is to consider MFI values above 1000 significant. In the field of HSCT, patients who were recipients of $\mathrm{T}$ cell depleted haploidentical transplants had a high incidence of graft failure with MFI $>1500$ and those that had T cell replete haploidentical transplants did not engraft with MFI $>500 ., 5$ A value above 5000 MFI has a very high likelihood of causing a rejection and therefore may be considered a relatively strong contraindication to the use of the donor against whom the antibodies are present unless strategies are utilized to reduce DSAs to acceptable levels. In case of cord blood transplant a value above $\mathrm{MFI}>1000$ already carries a higher risk for graft rejection and therefore more caution is needed.?

Studies have shown that up to $20-40 \%$ patients may harbor HLA specific antibodies. Nearly $24 \%$ of patients may have DSAs. In case of haploidentical transplants nearly $14.5 \%$ of patients may have DSAs to one or more HLA antigens. ${ }^{8}$ In another study it was noted that 3 of 4 patients, who underwent haploidentical transplants, failed to engraft owing to high titres of DSAs, this being the only significant factor that leads to graft failure compared to 1 of 20patients without DSAs. ${ }^{3}$

While using a different HSC donor, against whose HLA's the patient does not exhibit DSA's can be considered, it might not be possible, either because that is the only donor that is available or because most patients might not be able to wait longer secondary to disease- or patient-related factors. Therefore certain strategies have to be devised in order aid in these situations and to overcome the problem of increased risk for graft rejection. DSA production is mediated by B cells secondary to antigen exposure, resulting in the development of memory B cells and plasma cells. Therefore it stands to reason to target the DASs, B cells and their helpers namely the T cells, for which the following strategies have been deployed. ${ }^{4,9}$

i. Antibodies can be rendered less effective in mediating rejection when IVIG is given, as IVIG possibly modifies antibody levels through induction of anti-idiotypic circuits, inhibit complement activity and modulation of cytokine and immune cell activation, result in suppression of antigen presenting cell(APC) and B cell function, can promote B cell apoptosis and are potentially endothelial protective. ${ }^{10}$ 
ii. DSA numbers can be reduced by plasmapheresis. However both of these approaches result in short periods of response and have to be utilized very near to transplant and can also be repeated post-transplant but are resource intensive, especially plasmapheresis.

iii. B cells can be reduced by using rituximab with the caveat that it does not affect plasma cells. There is some concern that the agent might not be effective on its own since it does not affect already preformed antibodies. Therefore rituximab should be used in conjunction with other strategies.

iv. T cell depletion utilizing Anti-Thymocyte globulin (ATG), Mycophenolatemofetil (MMF), steroids and calcineurin inhibitors. ATG can impact B cells through mechanisms including the resulting decreased interaction between $\mathrm{CD} 4$ helper cells and B cells, thus decreasing B cell activation, B cell apoptosis and direct B cell cytoxicity leading to decrease in antibody production. ${ }^{9}$

v. Plasma cells: Bortezomib has been utilized in lieu of its potential effect on plasma cells. However, experience with bortezomib is lacking and there is some concern that the onset of its effects might take longer (about 1- to 1.5months) and therefore its exact place in current algorithms utilized to reduce DSA needs to be determined. At this point it is being used mostly in refractory cases. ${ }^{9}$

vi. Complement has been noted to play a role in antibody mediated rejection in solid organ transplantation, both via $\mathrm{C} 3 \mathrm{~b}, \mathrm{C} 4 \mathrm{~b}$, and $\mathrm{C} 5 \mathrm{~b}-\mathrm{C} 9$ (MAC). Eculizumab is a humanized monoclonal antibody that is effective against $\mathrm{C} 5$ which is important in terminal activation of complement. Patients who received eculizumab after kidney transplant had a lower incidence of rejection, yet the experience with this agent is overall limited. In one report, three patients who received eculizumab along with plasmapheresis and IVIG did not experience graft rejection after renal transplant during 1year of follow up. However, this remains a strategy whose efficacy is yet to be fully determined besides the expense associated with it could be prohibitive. ${ }^{11}$

vii. Splenectomy could potentially decrease antibody production by removal of a sizable body of B cells. It may prove to be effective in conjunction with other approaches detailed above but carries with it the lifelong risk of sepsis. Therefore, B cell directed therapies i.e. rituximab are increasingly replacing this approach, ${ }^{12}$

viii. However, none of the approaches in themselves are considered absolutely effective in reducing the DSA to a level that could be considered safe and timely. Therefore different combination strategies have been instituted to tackle this issue.

Johns Hopkins deducted an HSCT approach from its SOT experience and utilized a combination of Cytomegalovirus specific (CMV) IVIG, plasmapheresis, tacrolimus and MMF before HSCT to reduce the concentration of DSA. They reported that 8 of 9patients with high titres of DSA underwent this protocol and successfully engrafted. ${ }^{13}$ Other institutions have utilized a combination of rituximab, IVIG for prevention of graft rejection. ${ }^{14}$ However, these results for HSCT alone have not been published. Furthermore none of these approaches have been studied in a randomized fashion and therefore their comparative efficacy cannot be determined. In conclusion, while a better immunologic understanding has allowed to expand our armamentarium to combat DSA -mediated allograft rejection, multi-pronged strategies to improve outcome in these high risk HSCTs are currently being developed, as the battle has not yet been globally won.

\section{Acknowledgements}

None.

\section{Conflict of interest}

The author declares no conflict of interest.

\section{References}

1. Mattsson J, Ringden O, Storb R. Graft failure after allogeneic hematopoietic cell transplantation. Biol Blood Marrow Transplant. 2008;14(1):165-170.

2. Taylor PA, Ehrhardt MJ, Roforth MM, et al. Preformed antibody, not primed T cells, is the initial and major barrier to bone marrow engraftment in allosensitized recipients. Blood. 2007;109(3):1307-1315.

3. Ciurea SO, de Lima M, Cano P, et al. High risk of graft failure in patients with anti-HLA antibodies undergoing haploidentical stem-cell transplantation. Transplantation. 2009;88(8):1019-1024.

4. Takemoto SK, Zeevi A, Feng S, et al. National conference to assess antibody-mediated rejection in solid organ transplantation. Am J Transplant. 2004;4(7):1033-1041.

5. Ciurea SO, Mulanovich V, Saliba RM, et al. Improved early outcomes using a $\mathrm{T}$ cell replete graft compared with $\mathrm{T}$ cell depleted haploidentical hematopoietic stem cell transplantation. Biol Blood Marrow Transplant. 2012;18(12):1835-1844.

6. Yoshihara S, Maruya E, Taniguchi K, et al. Risk and prevention of graft failure in patients with preexisting donor-specific HLA antibodies undergoing unmanipulated haploidentical SCT. Bone Marrow Transplant. 2012;47(4):508-515.

7. Takanashi M, Atsuta Y, Fujiwara K, et al. The impact of antiHLA antibodies on unrelated cord blood transplantations. Blood. 2010;116(15):2839-2846.

8. Leffell MS, Jones RJ, Gladstone DE. Donor HLA-specific Abs: to BMT or not to BMT? Bone Marrow Transplant. 2015;50(6):751-758.

9. Levine $\mathrm{MH}, \mathrm{Abt}$ PL. Treatment options and strategies for antibody mediated rejection after renal transplantation. Semin Immunol. 2012;24(2):136-142.

10. Jordan SC, Vo A, Tyan D, Toyota M. Desensitization therapy with intravenous gammaglobulin (IVIG): applications in solid organ transplantation. Trans Am Clin Climatol Assoc. 2006;117:199-211.

11. Stegall MD, Diwan T, Raghavaiah S, et al. Terminal complement inhibition decreases antibody-mediated rejection in sensitized renal transplant recipients. Am J Transplant. 2011;11(11):2405-2413.

12. Kahwaji J, Vo AA, Jordan SC. ABO blood group incompatibility: a diminishing barrier to successful kidney transplantation? Expert Rev Clin Immunol. 2010;6(6):893-900.

13. Gladstone DE, Zachary AA, Fuchs EJ, et al. Partially mismatched transplantation and human leukocyte antigen donor-specific antibodies. Biol Blood Marrow Transplant. 2013;19(4):647-652.

14. Tanabe K, Inui M. Desensitization for prevention of chronic antibodymediated rejection after kidney transplantation. Clin Transplant. $2013 ; 27: 2-8$ 